\title{
Spatial discretization error in an artificial benchmark model of oblique laser incidence by finite volume approximation for radiative heat transfer
}

\author{
ZHANG HaoChun ${ }^{1,2} \&$ LI Yao ${ }^{2 *}$ \\ ${ }^{1}$ School of Energy Science and Engineering, Harbin Institute of Technology, Harbin 150001, China; \\ ${ }^{2}$ Institute of Composite Materials, Harbin Institute of Technology, Harbin 150001, China
}

Received October 14, 2011; accepted December 26, 2011

\begin{abstract}
The spatial discretization error in a finite volume method approximation for radiative heat transfer is investigated. An artificial benchmark model for oblique laser incidence on a two-dimensional rectangle containing a semi-transparent medium is proposed, in addition to using reference data from the Monte Carlo method. Within the framework of the current model, it is shown that numerical scattering in the finite volume method is affected by the spatial grid values and the different spatial discretization schemes to a large degree. Numerical scattering also varies with the degree of absorption coefficient deviation. Numerical scattering is distributed in a symmetrical profile along the laser incidence direction, and all of the schemes show symmetrical crossscattering.
\end{abstract}

finite volume method, spatial discretization error, radiative heat transfer, oblique laser incidence

Citation: Zhang H C, Li Y. Spatial discretization error in an artificial benchmark model of oblique laser incidence by finite volume approximation for radiative heat transfer. Chin Sci Bull, 2012, 57: 2046-2050, doi: 10.1007/s11434-012-5060-z

Radiative heat transfer is one of the main routes for thermal energy transport, especially in high temperature applications, and covers various problems from nature to engineering [1-3]. Fundamental approaches to investigate thermal radiation can be divided into three main categories: analytical, experimental and numerical. With the advances in computer and information technology, numerical methods have made great progress, and have eventually become the major research tool, with many approximation methods having been developed [4].

Among the numerical algorithms used for thermal radiation, the finite volume method (FVM), or finite volume approximation, has proven to be an efficient algorithm, with satisfactory precision shown in many applications $[5,6]$. However, as a numerical method, the FVM will essentially encounter different kinds of errors during the solution procedure. Because algebraic equations for the FVM are de-

*Corresponding author (email: liyao@hit.edu.cn) termined through discretization of the radiative transfer equation (RTE) over a specified control volume and a certain control angle, it inevitably suffers from discretization errors. The most important discretization errors occurring in the FVM are called the ray effect and false scattering, which were initially identified by Chai et al. [7].

Theoretical analysis and quantitative computation of the error, i.e., how precise the method can be, are integral aspects of the FVM [8]. This paper discusses only the error caused by spatial discretization, which is often referred as false scattering or numerical scattering [9]. Previous studies have found that numerical scattering is influenced by many factors, including the spatial discretization scheme [10], the grid resolution [11], the radiative properties [9] and the volumetric heat sources [12]. However, there are quite a few effective models for analysis and evaluation of the spatial discretization error.

In this work, an artificial benchmark model of oblique laser incidence on a two-dimensional (2D) rectangle con- 
taining a semi-transparent medium is proposed to investigate the numerical scattering, along with the use of reference data from the Monte Carlo method (MCM), which has been proven to generate no false scattering [13]. Within the framework of the current model, the effects of the spatial differential scheme, the spatial grid number and the absorption coefficient deviation of the medium on numerical scattering in the FVM are presented.

\section{Artificial benchmark model}

Consider a 2D rectangle containing an absorbing-emitting gray medium, in which the scattering coefficient $\kappa_{s}=0$; its refractive index is uniform, and is equal to that of the surroundings. In real cases, the medium may be gaseous, solid or liquid. However, in the current work, a generalized version of the participating medium is used. The four interfaces of the medium are all diffusely reflective, opaque and gray, i.e., transmittance $\gamma=0$. Both the northeast and southwest corners are coated with a thin film, and at a wavelength of $10.6 \mu \mathrm{m}$, the southwest corner is a semi-transparent and specularly reflective surface, i.e., the absorption coefficient $\alpha_{\lambda 10.6 \mu \mathrm{m}}=0$ and $\rho_{\lambda=10.6 \mu \mathrm{m}}^{s}+\gamma_{\lambda=10.6 \mu \mathrm{m}}=1$. At other wavelengths $(\lambda \notin 10.6 \mu \mathrm{m})$, it is a diffusely reflective, opaque and gray surface $\left(\gamma_{\lambda \notin 10.6 \mu \mathrm{m}}=0\right)$. The thin film in the northeast corner is opaque, and is a diffusely reflective surface, except at the wavelength of $10.6 \mu \mathrm{m}$. A laser beam at the wavelength of $10.6 \mu \mathrm{m}$ is projected from the southwest corner to the northeast corner (the shadowed area), as shown in Figure 1.

First, the laser beam is projected from the southwest corner. Because there is no scattering in the medium, and the refractive index is uniform, there is no refraction of the laser beam. When the beam arrives at the northeast corner,

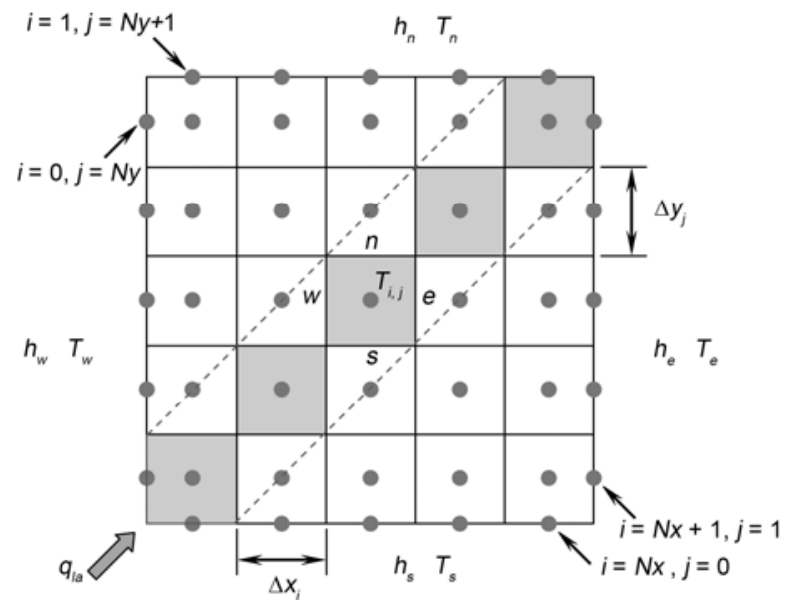

Figure 1 Artificial model of oblique laser incidence on a 2D participating medium. it is specularly reflected in the opposite direction without angle deviation. In terms of the actual physical process, there is no scattering during laser propagation through the semi-transparent medium. Within a finite time, the temperature will increase only in the laser-irradiated region. However, in the FVM simulation of the laser incidence, numerical scattering can appear. If the scattering phenomenon occurs, i.e., the temperature increases in the non-incident region (i.e. the region which is not irradiated by the laser), it means that false scattering can happen, and vice versa.

The radiative heat transfer process in the artificial model above and the detailed derivation of the governing equation can be found in [13].

\section{Brief introduction to numerical scattering}

Because numerical scattering is a multi-dimensional problem [13], a 2D case is taken into account in the current study, without considering the scattering in the medium.

The accurate solution for the spectral radiative intensity in a specific angular direction $\Omega^{l}$ of the FVM is denoted by $I_{s}^{\Omega^{l}}$, and the symbol $L(I)_{s, \Omega^{l}}$ is defined as an operator for the differential process of $I_{s}^{\Omega^{l}}$ at a control volume of $\left(s, \Omega^{l}\right)$, where $s$ denotes the spatial coordinates of the point, as shown in eq. (1):

$$
L(I)_{s, \Omega^{l}}=\left[\xi \frac{\partial I}{\partial x}+\eta \frac{\partial I}{\partial y}-\kappa_{\alpha \lambda} I-S\right]_{s, \Omega^{l}} .
$$

If the symbol $L_{\Delta s}\left[I_{s}^{\Omega^{l}}\right]$ is used for the difference operator for $I_{s}^{\Omega^{l}}$, we then have:

$$
L_{\Delta s}\left[I_{s}^{\Omega^{l}}\right]=\xi \frac{I_{i+1}^{\Omega^{l}}-I_{i}^{\Omega^{l}}}{\Delta x}+\eta \frac{I_{j+1}^{\Omega^{l}}-I_{j}^{\Omega^{l}}}{\Delta y}-\kappa_{\alpha \lambda} I^{\Omega^{l}}-S_{s}^{\Omega^{l}}
$$

In eq. (2), $L_{\Delta s}\left[I_{s}^{\Omega^{l}}\right]$ denotes the step scheme for a $2 \mathrm{D}$ differential equation. In this way, the cut-off for the discrete differential equations denotes the difference between the difference operators and the corresponding differential operators, which can be expressed using $T E$, i.e.

$$
T E=L_{\Delta s}\left[I_{s}^{\Omega^{l}}\right]-L(I)_{s, \Omega^{l}}
$$

Eq. (3) can be deduced using the Taylor expansion of the difference equation. For the case above, the Taylor expansions of $I_{\boldsymbol{s}}^{\Omega^{l}}$ and $I_{\boldsymbol{s}+d \boldsymbol{s}}^{\Omega^{l}}$ in the space position $\boldsymbol{s}=\boldsymbol{s}(i, j)$ can be substituted into the difference equation and reorganized, and we then obtain the correlation function shown 
in eq. (4):

$$
T E=O\left(\Delta x^{2}, \Delta y^{2}\right)+\left(S_{s}^{\Omega^{l}}-\left.S\right|_{s, \Omega^{l}}\right) .
$$

For a 2D problem, if the derivative is substituted for a first-order difference, if the radiative intensity profile is assumed by the space differential schemes, and if the transfer direction intersects with the grid and a non-negative radiative intensity gradient exists in the direction perpendicular to the transportation direction, numerical scattering is still generated.

Generally, a relationship should be derived to correlate the radiative intensities in the face of the control volume and the intensities at the center of the control volume, which constitute a kind of spatial differential scheme. In view of this, the numerical scattering is also related to the spatial differential factor, which is shown in eq. (5):

$$
T E=\left|\frac{\partial I^{\Omega^{l}}}{\partial x}-\frac{I_{e}^{\Omega^{l}}-I_{w}^{\Omega^{l}}}{\Delta x}\right|=\left|\frac{\left(1-2 f_{x}\right) \Delta x}{2 !}\left(\frac{\partial^{2} I^{\Omega^{l}}}{\partial x^{2}}\right)\right| .
$$

In a multi-dimensional problem, the numerical scattering acts in a similar manner to the way it acted in one dimension.

In the steady state case, the temperature can be determined by the following correlation, in which:

$$
\begin{aligned}
\nabla \cdot \boldsymbol{q}^{r} & =\sum_{k=1}^{M_{b}} \kappa_{k}\left(4 \pi I_{b k}-H_{k}\right) \\
& =\sum_{k=1}^{M_{b}} \kappa_{k}\left[4 B_{k, T_{P}} \sigma T_{P}^{4}-\sum_{\Omega^{m} \in 4 \pi} I_{k}^{\Omega^{l}} \Omega^{\Omega^{l}}\right] .
\end{aligned}
$$

Also, the fact that the spatial discretization error can be reduced only by increasing the grid numbers is not a simple problem. From eqs. (1)-(6), the radiative intensity $I$ varies with the wavelength $\lambda$, based on the assumption of spectral band consistency, which affects the radiative property, i.e., $\kappa_{\alpha \lambda}$. Therefore, errors caused by the radiative property are also included in the numerical scattering within the scope. For the approximation complexity of the spectral absorption coefficient, a ratio $\chi$ is applied to denote its deviation, which is shown in eq. (7):

$$
\kappa_{\alpha \lambda}^{*}=\chi \kappa_{\alpha \lambda} .
$$

To summarize, the factors that affect $T E$ can be implemented in a function, which is shown in eq. (8), and it is almost the same as in [9], which can be similarly extended to a three-dimensional problem.

$$
T E=T E\left[(\Delta x, \Delta y), f_{x, y}, \kappa_{\alpha \lambda}\right] .
$$

\section{Simulation results and analysis}

The radiative properties and computing parameters are: the geometry of the computing domain, $L_{x}=L_{y}=0.25 \mathrm{~m}$; the refractive index of the medium $n=1$; the absorption coefficient $\kappa_{\alpha \lambda}=1 \mathrm{~m}^{-1}$; and the scattering coefficient $\kappa_{\mathrm{s} \lambda}=0$. The incident wavelength of the laser beam is $\lambda_{l a}=10.6 \mu \mathrm{m}$, and the energy flux of the laser beam is $q_{l a}=2 \times 10^{3} \mathrm{~kW}$. Because numerical scattering does not exist in the MCM [13], the results of MCM can be used as a benchmark solution to test FVM accuracy. In the FVM, the solid angle is divided into $N \theta \times$ $N \varphi=20 \times 28$. Also, the thermal conductivity of this medium is specified to be extremely small, i.e., $k=0.76545 \times 10^{-50}$ $\mathrm{W} /(\mathrm{m} \mathrm{K})$, and therefore, thermal radiation is the dominant heat transfer method.

To analyze and compare the results, the following expression for the temperature increments is defined, where $T_{0}$ $=1000 \mathrm{~K}$ is the initial temperature:

$$
\Delta T_{i, j(\text { or } m, n)}=T_{i, j(\text { or } m, n)}-T_{0} .
$$

The ratio $E r_{l a}$ is defined in the following as the ratio of the maximum temperature increment where the node is outside the laser incidence region to the minimum temperature increment where the node is within the laser incidence region, i.e.,

$$
E r_{l a}=\frac{\max \left(\Delta T_{i, j}\right)(i, j \notin \text { Laser incidence })}{\min \left(\Delta T_{m, n}\right)(m, n \in \text { Laser incidence })} .
$$

$E r_{l a}$ is applied to compare numerical scattering values, and values for different schemes with different grid numbers are shown in Table 1 for the step scheme (FVM1), the diamond scheme (FVM2) and the exponential scheme (FVM3).

To clarify the effects of the numerical scattering, contours of the temperature profiles for the different methods are shown in Figures 2-5.

It can be seen from these tables and figures that when the grid number increases, numerical scattering is reduced. This is the same tendency as in all other fields. However, the accuracy of the FVMs is also affected by the space differential scheme, and among these schemes, the diamond scheme has the highest accuracy, and the exponential scheme is less accurate, while the step scheme has the lowest accuracy of the three schemes. Conversely, the degree of numerical scattering follows the reverse order, i.e., the step scheme produces minimum numerical scattering, and the exponential

Table $1 E r_{l a}$ for temperature profile of oblique laser incidence by MCM and FVM (FVM1 for step scheme, FVM2 for diamond scheme and FVM3 for exponential scheme)

\begin{tabular}{ccccc}
\hline & FVM1 (\%) & FVM2 (\%) & FVM3 (\%) & MCM (\%) \\
\hline$N x \times N y=5 \times 5 E r_{l a}$ & 6.155 & 11.607 & 7.589 & 0.064 \\
$N x \times N y=10 \times 10 E r_{l a}$ & 4.004 & 7.859 & 5.019 & 0.043 \\
$N x \times N y=20 \times 20 E r_{l a}$ & 2.395 & 4.789 & 3.025 & 0.026 \\
\hline
\end{tabular}




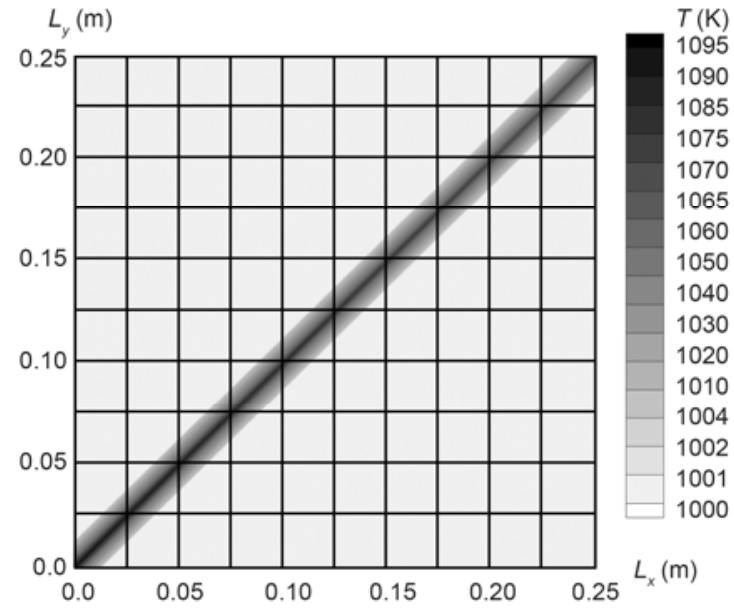

Figure 2 Temperature contour by MCM.

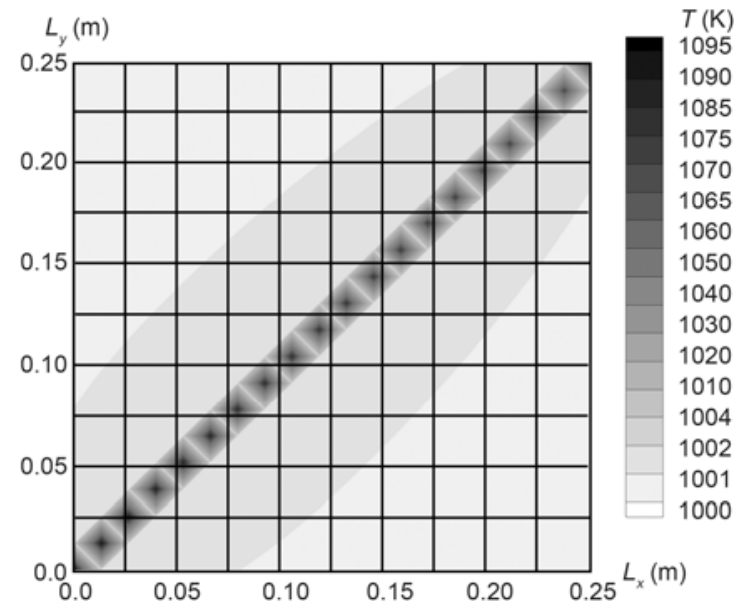

Figure 3 Temperature contour by FVM1 (for step scheme).

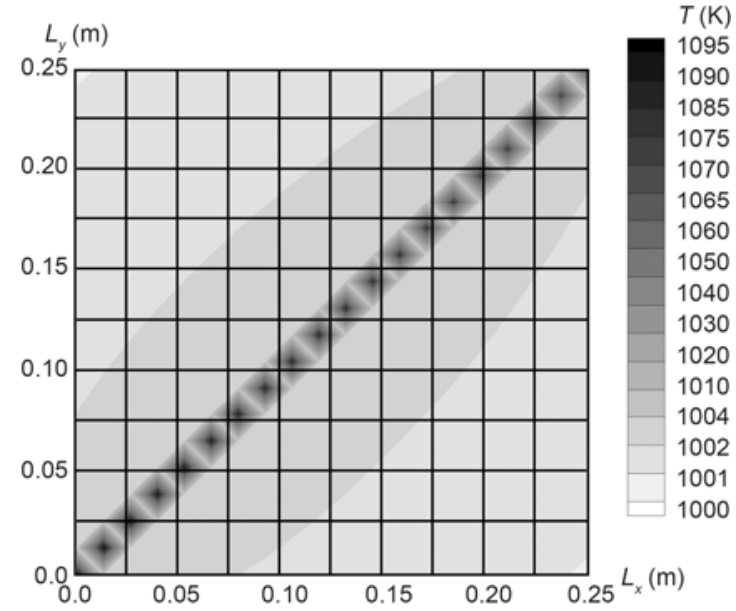

Figure 4 Temperature contour by FVM2 (for diamond scheme).

scheme produces more, while the diamond scheme produces the maximum scattering of the three methods.

Choosing the $x=0.125$ position and for different heights,

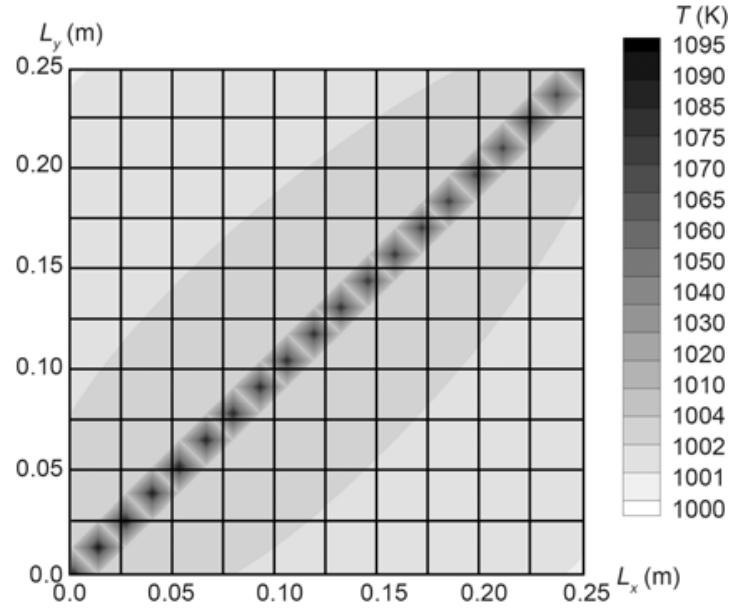

Figure 5 Temperature contour by FVM3 (for exponential scheme).

the distributions of the numerical scattering for different spatial differential schemes with grid numbers $N x \times N y=5 \times 5$ are shown in Figure 6. If we set the direction of laser incidence as the central axis, we see that the numerical scattering is distributed symmetrically along the axis, which is called symmetrical cross-scattering. All three schemes show symmetrical cross-scattering.

By considering the individual absorption coefficients $\kappa_{\alpha \lambda}^{*}$ of $0.1,1.0$, and 2.0 , and the corresponding optical thicknesses $\tau=0.025,0.25,0.5$, and 2.5 , the numerical test results for different grid numbers for the MCM and FVM1 are shown in Table 2. We can see that when the absorption coefficient deviation is high, the numerical scattering cannot be eliminated by increasing the grid number alone.

\section{Conclusion}

An artificial benchmark model of oblique laser incidence on

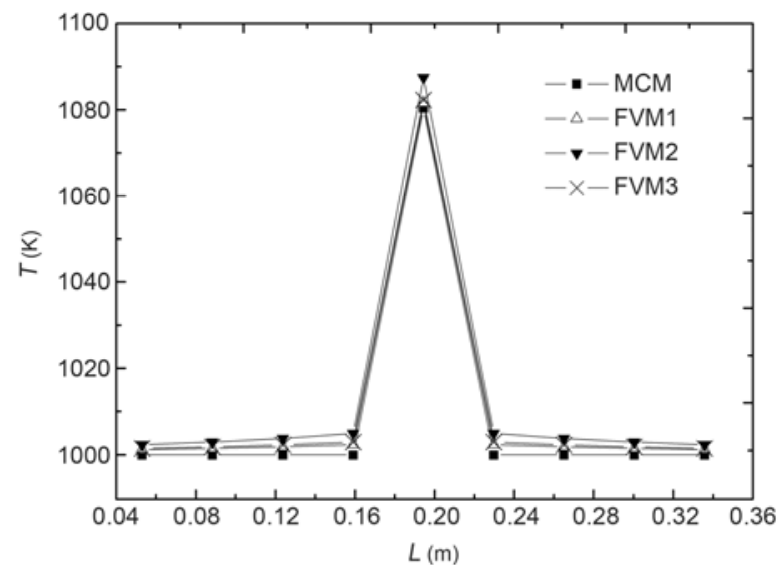

Figure 6 Temperature profile of oblique laser incidence by MCM and FVM (FVM1 for step scheme, FVM2 for diamond scheme and FVM3 for exponential scheme). 
Table $2 E r_{l a}$ for MCMs and FVMs of different absorption coefficients

\begin{tabular}{cccc}
\hline$N x \times N y$ & $\kappa_{\alpha \lambda}$ & $E r_{l a} \rightarrow \mathrm{MCM}$ & $E r_{l a} \rightarrow \mathrm{FVM}$ \\
\hline \multirow{3}{*}{$5 \times 5$} & 0.1 & 0.0 & 0.613 \\
& 1.0 & 0.054 & 7.164 \\
& 2.0 & 0.282 & 15.132 \\
\hline \multirow{3}{*}{$20 \times 20$} & 0.1 & 0.0 & 0.191 \\
& 1.0 & 0.026 & 2.395 \\
& 2.0 & 0.127 & 6.166 \\
\hline
\end{tabular}

a $2 \mathrm{D}$ rectangle containing a semi-transparent medium is proposed to investigate the numerical scattering in the FVM, along with the use of reference data from the MCM, which has been proven to generate no false scattering.

Within the framework of the current model, it is shown that numerical scattering for the FVM is affected by the spatial grid values and is also affected by the different spatial discretization schemes to a large degree, with the diamond scheme being best, then the exponential scheme and finally the step scheme, in ranked order. Numerical scattering also varies with the amount of absorption coefficient deviation. When the absorption bias is high, the numerical scattering cannot be eliminated solely by increasing the grid number. Also, numerical scattering is distributed symmetrically along the laser incidence direction, and all of the schemes show symmetrical cross-scattering.

This work was supported by the National Natural Science Foundation of China (51121004, 51006026, 90916020) and the Development Program for Outstanding Young Teachers in Harbin Institute of Technology (HITQNJS.2009.022).
1 Howell J R, Siegel R, Menguc M P. Thermal Radiation Heat Transfer. 5th ed. New York: CRC Press, 2010

2 Cui Y, Huang Y, Li W, et al. TM polarization characteristics on thermal radiation of a negative refractive index thin film. Chin Sci Bull, 2009, 54: 1663-1668

3 Zheng Z H, Xuan Y M. Near-field radiative heat transfer between general materials and metamaterials. Chin Sci Bull, 2011, 56: 23122319

4 Tan H P, Liu L H, Yi H L, et al. Recent progress in computational thermal radiative transfer. Chin Sci Bull, 2009, 54: 2627-2637

5 Daniel R R, Fatmir A. A consistent interpolation function for the solution of radiative transfer on triangular meshes. I-Comprehensive formulation. Numer Heat Transfer Part A, 2011, 59: 97-115

6 Das R, Mishra S C, Ajith M, et al. An inverse analysis of a transient 2-D conduction-radiation problem using the Lattice Boltzmann method and the finite volume method coupled with the genetic algorithm. J Quant Spectrosc Radiat Transfer, 2008, 109: 2060-2077

7 Chai J C, Lee H S, Patankar S V. Ray effect and false scattering in the discrete ordinates method. Numer Heat Transfer Part B, 1993, 24: 373-389

8 Raithby G D, Chui E H. A finite-volume method for predicting a radiant heat transfer in enclosures with participating media. J Heat Transfer, 1990, 112: 415-423

9 Zhang H C, Tan H P. Evaluation of numerical scattering in finite volume method for solving radiative transfer equation by a central laser incidence model. J Quant Spectrosc Radiat Transfer, 2009, 110: 1965-1977

10 Coelho P J. A comparison of spatial discretization schemes for differential solution methods of the radiative transfer equation. J Quant Spectrosc Radiat Transfer, 2008, 109: 189-200

11 Kallinderis Y, Kontzialis C. A priori mesh quality estimation via direct relation between truncation error and mesh distortion. J Comput Phys, 2009, 228: 881-902

12 Kamel G, Naceur B M, Rachid M, et al. Formulation and testing of the ftn finite volume method for radiation in 3-d complex inhomogeneous participating media. J Quant Spectrosc Radiat Transfer, 2006, 98: 425-445

13 Tan H P, Zhang H C, Zhen B. Estimation of ray effect and false scattering in approximate solution method for thermal radiative transfer equation. Numer Heat Transfer Part A, 2004, 46: 807-829

Open Access This article is distributed under the terms of the Creative Commons Attribution License which permits any use, distribution, and reproduction in any medium, provided the original author(s) and source are credited. 